\title{
Interpolation of Digital Elevation Models Using AMLE and Related Methods
}

\author{
Andrés Almansa, Frédéric Cao, Yann Gousseau, and Bernard Rougé
}

\begin{abstract}
Interpolation of digital elevation models becomes necessary in many situations, for instance, when constructing them from contour lines (available e.g., from nondigital cartography), or from disparity maps based on pairs of stereoscopic views, which often leaves large areas where point correspondences cannot be found reliably.

The absolutely minimizing Lipschitz extension (AMLE) model is singled out as the simplest interpolation method satisfying a set of natural requirements. In particular, a maximum principle is proven, which guarantees not to introduce unnatural oscillations which is a major problem with many classical methods. We then discuss the links between the AMLE and other existing methods. In particular, we show its relation with geodesic distance transformation. We also relate the AMLE to the thin-plate method, that can be obtained by a prolongation of the axiomatic arguments leading to the AMLE, and addresses the major disadvantage of the AMLE model, namely its inability to interpolate slopes as it does for values. Nevertheless, in order to interpolate slopes, we have to give up the maximum principle and authorize the appearance of oscillations. We also discuss the possible link between the AMLE and Kriging methods that are the most widely used in the geoscience literature. We end by numerical comparison between the different methods.
\end{abstract}

Index Terms-Digital elevation models, image interpolation, partial differential equations.

\section{INTRODUCTION}

$\mathbf{I}$ N THIS PAPER, we study the problem of interpolating twodimensional (2-D) data. This is motivated by several problems that may be encountered when trying to reconstruct digital elevation models

- For instance, for maps which are not yet available in digital form, the elevation is only known on a limited number of level curves, and we then have to interpolate in order to retrieve a three-dimensional (3-D) model of elevation.

- One way to construct elevation models is to find points of correspondence between two images from a stereo vision pair of a scene. Most existing matching algorithms fail on some parts of the image and no elevation can be retrieved [1]. Interpolation is then also necessary.

In both cases, we may assume that the elevation is regular in the areas where it is a priori unknown. In the first case, the lines

Manuscript received November 29, 2000; revised August 28, 2001.

A. Almansa and F. Cao are with the Centre de Mathémathiques et Leur Applications, École Normale Supérieure de Cachan, 94235 Cachan, France (e-mail: almansa@cmla.ens-cachan.fr; cao@cmla.ens-cachan.fr).

Y. Gousseau is with the Department Traitement du Signal et des Images, École Nationale des Telécommunications, 75634 Paris, France (e-mail: gousseau@tsi.enst.fr).

B. Rougé is with the Centre National d'Etudes Spatiales, 31055 Toulouse, France (e-mail: bernard.rouge@cnes.fr).

Publisher Item Identifier S 0196-2892(02)01874-0. whose level is known are in general chosen such that they are representative of the real elevation. Therefore, the elevation between two adjacent lines always lies between the corresponding levels. Moreover, it is not likely that oscillations between both lines can be significant, else some additional level lines would have also been represented. This implies that a reasonable interpolation method must not artificially create some oscillations between given level curves otherwise, it may create some arbitrary information which cannot be inferred from the data. In the second case, correlation is commonly used to find point correspondences. In this case, one of the major reasons why matching fails is that the variations of the image are not high enough in some region, which implies that the corresponding elevation is likely to be regular.

An interpolation model called absolutely minimizing Lipschitz extension (AMLE) was introduced in [2] as the most simple interpolant satisfying a set of natural axioms. We think that these axioms are also very well-suited for the interpolation of digital elevation models. We recall these axioms in Section II. A very nice property of the AMLE, that can be mathematically proved, is that it does not create any artifacts nor oscillations. This model is a real interpolation model that exactly fits the data. ${ }^{1}$ It can interpolate values on isolated curves and even isolated points (for instance mountain or hill top).

We show in Section III how the AMLE can be related to other methods already used for DEM interpolation. First, it can be viewed as the stationary state of iterated geodesic distance transformations (see Section III-A). Second, it may happen that the elevation is known not only on isolated points but on a domain and we want to reconstruct the elevation outside this set. In this case, we can take advantage of the value on the boundary of the domain but also of some higher order information such as the slope of the data. The AMLE cannot handle more than zeroth order information and neither do geodesic distance interpolators. The thin-plate model discussed in Section III-B can be viewed as an attempt to generalize the axiomatic approach leading to the AMLE. It allows to interpolate values as well as gradient fields on the boundary of the interpolation domain, but it does not guarantee to avoid oscillations as the AMLE model does. Kriging methods (see Section III-C) are also related to the thin-plate model in some cases, and we compare the AMLE, the thin-plate and kriging in Section IV where we show some interpolation results for methods. Finally in Section V we discuss some issues for future research.

\footnotetext{
${ }^{1}$ Here, we mean a model that actually fits the data on curves and isolated points, and does not have zero capacity for points like Laplace's operator for instance (see Section II for a discussion).
} 


\section{AMILE MODEL}

In this section, we introduce the AMLE model. It has been introduced in [2] as one of the pertinent models for image interpolation, though it had been previously studied from a completely theoretical point of view in [3], [4], and [5]. The approach they use is axiomatic, that is, from a small number of natural postulates, they derive a classification of interpolation operators. Among the ones satisfying the largest number of these natural properties, the AMLE model will be particularly relevant in the problems we are interested in. In particular, it will be very efficient to interpolate data between level lines. This shall be interesting for elevation reconstruction from a scanned map where only a few level lines (iso-level curves) are available. We shall also use it to interpolate scattered data obtained from a correlation between stereo pairs of a scene.

Let us now give the main ideas leading to the AMLE model. We assume that the elevation we want to interpolate is the interior of a domain $\Omega$ in the plane. We assume that we only know the value $\varphi$ of elevation on $\partial \Omega$, the boundary of $\Omega$. Note that this is realistic in the interpolation of iso-level lines. We assume that it is possible to interpolate $\varphi$ inside $\Omega$. Let us denote by $E(\Omega, \varphi): \Omega \rightarrow \mathbb{R}$ the obtained elevation. We now review some natural properties that $E(\Omega, \varphi)$ should satisfy.

(P1) The interpolation is stable, that is to say, if we interpolate the values of $E(\Omega, \varphi)$ on a subdomain of $\Omega$, the result does not change. More precisely, for any subdomain $\Omega^{\prime} \subset \Omega$, we have

$$
E\left(\Omega^{\prime}, E(\Omega, \varphi)_{\mid \partial \Omega^{\prime}}\right)=E(\Omega, \varphi)_{\mid \Omega^{\prime}}
$$

where the subscript $\mid A$ designs the restriction of a function to the set $A$.

(P2) The interpolation respects the global elevation ordering, that is

$$
\text { if } \varphi \leq \psi \text { on } \partial \Omega \text {, then } E(\Omega, \varphi) \leq E(\Omega, \psi) \text {. }
$$

(P3) Finally, we give a more technical property giving the behavior of $E$ on second order polynomials. It consists in assuming the existence of a function $F$ such that if $u(x)=$ $c+\left(p, x-x_{0}\right)+1 / 2\left(A\left(x-x_{0}\right),\left(x-x_{o}\right)\right)$, then

$$
\lim _{r \rightarrow 0} \frac{E\left(u, u_{\mid D\left(x_{0}, r\right)}\right)\left(x_{0}\right)-u\left(x_{0}\right)}{r^{2} / 2}=F\left(A, p, c, x_{0}\right) .
$$

To this three basic axioms, we add a set of natural geometric properties.

(P4) The interpolation does not depend on the position of $\Omega$. In other terms, it commutes with translation.

(P5) It does not depend on the orientation of $\Omega$ either. Put another way, it commutes with plane rotations.

(P6) It is also scale invariant (i.e., commutes with dilations).

(P7) The reference level is arbitrary, hence the interpolation must commute with the addition of a fixed value.

(P8) The elevation unit is also arbitrary. Therefore, the interpolation has to commute with multiplication by a fixed constant.
Before we give the general form of an interpolation operator satisfying these properties, let us give some notations. If $u: \Omega \subset$ $\mathbb{R}^{2} \rightarrow \mathbb{R}$, the gradient of $u$ denoted by $D u$, is the vector with coordinates $\left(u_{x}, u_{y}\right)$ of the partial derivatives of $\mathrm{u}$ with respect to $\mathrm{x}$ and $\mathrm{y}$. We denote by $D u^{\perp}$, the vector obtained from $D u$ by a rotation of angle $\pi / 2$ [the orientation does not matter; we arbitrarily chose it such that $\left(D u, D u^{\perp}\right)$ is counterclockwise if $D u \neq 0$ ]. We also denote by $D^{2} u$ the Hessian matrix of $u$, that is to say, the symmetric matrix whose coefficients are the second derivatives of u. As usual, we canonically associate a quadratic form to $D^{2} u$. We also use the notation

$$
u_{\xi \xi}=D^{2} u\left(\frac{D u}{|D u|}, \frac{D u}{|D u|}\right)
$$

to represent the second derivative of $u$ in the direction of the gradient, and similarly

$$
u_{\eta \eta}=D^{2} u\left(\frac{D u^{\perp}}{|D u|}, \frac{D u^{\perp}}{|D u|}\right)
$$

and

$$
u_{\eta \xi}=D^{2} u\left(\frac{D u^{\perp}}{|D u|}, \frac{D u}{|D u|}\right)
$$

to represent the second derivative of $u$ in the direction of the level curves, and the mixed derivative, respectively. The following result characterizes all interpolators satisfying the required axioms:

Theorem 1 [2]: Assume that $E(\Omega, \varphi)$ satisfies the properties (P1) $-(\mathbf{P 8})$. Then, it is a viscosity solution of the equation

$$
G\left(u_{\xi \xi}, u_{\eta \eta}, u_{\eta \xi}\right)=0
$$

where $G(a, b, c)$ is positively homogeneous of degree one and nondecreasing with respect to the matrix $\left(\begin{array}{ll}a & c \\ c & b\end{array}\right)$. If we assume that $G$ is differentiable at the point $(0,0,0)$, then, $G$ is linear, that is $G(a, b, c)=\alpha a+\beta b+\gamma c$ where $(\alpha, \beta, \gamma)$ satisfies the property $\alpha \beta-\gamma^{2} \geq 0$.

The viscosity solution theory is the correct mathematical setting for nonlinear elliptic equations of the type (4). We refer the reader to [6] and [7] for complete details.

In the following, we shall assume that the function $G$ in (4) is differentiable, and, thus, linear. We see, that the most simple equations correspond to the case.

1) $G(a, b, c)=a+b$. In this case, the equation is $\Delta u=0$, that is Laplace equation.

2) $G(a, b, c)=a$, yielding the equation

$$
D^{2} u\left(\frac{D u}{|D u|}, \frac{D u}{|D u|}\right)=0
$$

which is Aronsson's AMLE model.

3) $G(a, b, c)=b$, yielding the equation

$$
D^{2} u\left(\frac{D u^{\perp}}{|D u|}, \frac{D u^{\perp}}{|D u|}\right)=0 .
$$

This equation means that the curvature of the iso-level lines equals zero. As a consequence, these curves are straight lines.

Laplace equation is well known for regular interpolation since it can describe the equilibrium temperature distribution in a do- 
main with source on the boundary. Nevertheless, by classical results of potential theory, this model does not allow to fix values at isolated points, and we say that it has zero capacity for points. For instance, if $\Omega=D(0,1) \backslash\{0\}$ in the plane (the pointed unit disk) and if we fix $\varphi=0$ on the unit circle and $\varphi=1$ at the origin, the solution of Laplace equation is identically zero, letting the value at the origin ignored! It is possible to prove that any linear combination of the form

$$
\alpha D^{2} u\left(\frac{D u}{|D u|}, \frac{D u}{|D u|}\right)+\beta D^{2} u\left(\frac{D u^{\perp}}{|D u|}, \frac{D u^{\perp}}{|D u|}\right)
$$

with positive $\alpha$ and $\beta$ satisfies the same undesirable property.

The equation

$$
D^{2} u\left(\frac{D u^{\perp}}{|D u|}, \frac{D u^{\perp}}{|D u|}\right)=0
$$

may not have a unique solution. In [8], Masnou and Morel found a way to choose the best solution by introducing a variational condition. This algorithm, called disocclusion gives impressive results in restoring images, but the domain $\Omega$ must be simply connected (which is a too strong condition for our application) and the fact that iso-level curves are straight curves somehow gives irrealistic elevation models.

On the contrary, the AMLE model can interpolate isolated values. In the case of the pointed disk, the solution is the one we can expect, that is $u(x)=1-|x|$. The AMLE model is mathematically well posed as exposed in the following theorem.

Theorem 2 [3] and [5]: Let $\Omega$ be a bounded domain. Assume that $\varphi$ is continuous on $\partial \Omega$. Then, there exists a unique viscosity solution of

$$
D^{2} u\left(\frac{D u}{|D u|}, \frac{D u}{|D u|}\right)=0
$$

with boundary value equal to $\varphi$. Moreover, the AMLE satisfies the properties $(\mathbf{P 1})-(\mathbf{P 8})$.

(A generalization of this result, which relaxes the continuity assumption on the boundary data $\varphi$, was proven in [9].)

The reason why this model is named AMLE is given by the following proposition.

Theorem 3 [5]: Let $\Omega$ be a bounded domain and $\varphi$ be a Lipschitz function on $\partial \Omega$. Then the AMLE is the unique function $\mathrm{u}$ interpolating $\varphi$ and satisfying the property

$\forall \Omega^{\prime} \subset \Omega, \quad \forall w$ Lipschitz in $\partial \Omega^{\prime}, w=u$ on $\partial \Omega^{\prime} \Rightarrow$

$$
\|D u\|_{\infty} \leq\|D w\|_{\infty}
$$

This means that the AMLE minimizes the Lipschitz constant on any subdomain, which is an important property when interpolating elevation models because it is a way to ensure that the interpolation will not create information that is not present in the original data.

Many interpolation methods may produce oscillations (Gibbs effects, overshoots) which are not apparent from the original data. Such oscillations appear rather as a byproduct of regularity assumptions which do not match the given data. Theorem 3 ensures that the AMLE interpolant cannot create such oscillations.
Section IV shows some experimental results using this model, and in Appendix A we give some details on the numerical solution of this equation and its computational complexity.

\section{RELATIONS TO PREVIOUS WORK ON DEM INTERPOLATION}

Whereas the AMLE model has been recently proposed for image processing in [2], it is essentially new in the geoscience domain. In this section we explore the close relationships between AMLE and other classical methods that have been proposed in the geoscience literature.

\section{A. Geodesic Distance Transformations}

Interpolators based on geodesic distances are to the best of our knowledge the main tool for constructing DEMs from contour lines, and fast algorithms have been proposed in [10] and [11]. Starting from the assumption that the boundary can be partitioned into two disjoint regions $\partial \Omega=\partial \Omega_{1} \cup \partial \Omega_{2}$, such that the boundary conditions are constant in each of the regions, i.e., $\left.u\right|_{\partial \Omega_{1}}=c_{1} \in \mathbb{R}$ and $\left.u\right|_{\partial \Omega_{2}}=c_{2} \in \mathbb{R}$, the geodesic distance interpolator can be defined as

$$
u(x)=\frac{c_{2} d_{\Omega, g}\left(x, \partial \Omega_{1}\right)+c_{1} d_{\Omega, g}\left(x, \partial \Omega_{2}\right)}{d_{\Omega, g}\left(x, \partial \Omega_{1}\right)+d_{\Omega, g}\left(x, \partial \Omega_{2}\right)}
$$

where $d_{\Omega, g}(x, A)$ is the generalized geodesic distance from a point $x$ to a set A, i.e., the minimal usual geometric length of a path $C$ entirely contained in $\Omega$, which minimizes the geodesic distance $\int_{C} g$ among all paths joining $x$ to a point $y \in A$. Whereas in [10] a constant weighting function $g$ was used, in a later article [11] it was proposed to use the Euclidean distance to the border $g(x)=[d(x, \partial \Omega)]^{c}$ as a weighting function, in order to avoid certain artifacts occurring when geodesic paths $C$ become tangent to the border. ${ }^{2}$

This produces results which are very close to the AMLE model, which is not surprising since the AMLE model can heuristically be obtained as a fixed point of geodesic distance interpolations. In fact, assuming that e.g., $c_{1} \leq c_{2}$ consider the following iteration:

$$
\begin{aligned}
u_{0}(x)= & \frac{c_{2} d_{\Omega, 1}\left(x, \partial \Omega_{1}\right)+d_{\Omega, 1}\left(x, \partial \Omega_{2}\right) c_{1}}{d_{\Omega, 1}\left(x, \partial \Omega_{1}\right)+d_{\Omega, 1}\left(x, \partial \Omega_{2}\right)} \\
u_{n+1}(x)= & \frac{c_{2} d_{\Omega, u_{n}^{c}}\left(x, \partial \Omega_{1}\right)+d_{\Omega, u_{n}}\left(x, \partial \Omega_{2}\right) c_{1}}{d_{\Omega, u_{n}^{c}}\left(x, \partial \Omega_{1}\right)+d_{\Omega, u_{n 2}}\left(x, \partial \Omega_{2}\right)} \\
& \text { if } n \geq 0 .
\end{aligned}
$$

If the iteration converges to a fixed point

$$
u_{\infty}=\frac{c_{2} d_{\Omega, u_{\infty}^{c}}\left(x, \partial \Omega_{1}\right)+d_{\Omega, u_{\infty}}\left(x, \partial \Omega_{2}\right) c_{1}}{d_{\Omega, u_{\infty}^{c}}\left(x, \partial \Omega_{1}\right)+d_{\Omega, u_{\infty}}\left(x, \partial \Omega_{2}\right)}
$$

then $u_{\infty}$ is AMLE. Indeed, the geodesic paths associated to the potential $u_{\infty}$ are locally the gradient curves ${ }^{3}$ of $u_{\infty}$. Hence, equation (8) means that $u_{\infty}$ is linear along its own gradient curves, i.e., $\left\|D u_{\infty}\right\|$ is constant in the direction of $D u_{\infty}$, or put

${ }^{2}$ Here the complement operation is defined as $g^{c}=-g+\max _{\Omega} g$.

${ }^{3} \mathrm{~A}$ gradient curve of a function $\mathrm{u}$ is a curve such that its tangent vector is always parallel to $D u$. 
another way $\mathrm{u}_{\infty}$ satisfies the AMLE equation $D^{2} u(D u, D u)=$ 0 .

This result has two important implications: 1) the different variations of (noniterated) geodesic distance transform methods can be interpreted as truncated implementations of the AMLE model and 2) they can be used to write faster algorithms to solve the AMLE in the particular case of contour line interpolation.

\section{B. Thin Plate Model}

A major drawback of the AMLE model is that it cannot interpolate slopes, it can only interpolate boundary data. As we shall see later in this section and in Section IV, this does not always represent a serious problem, and there are many reasons for keeping the AMLE method despite this drawback. However, the fact that it cannot interpolate slopes may result in flat mountain tops and slope discontinuities across some level curves. This is not really serious, at the sides of a mountain or when looking at the DEM as a gray-level image, but it becomes sometimes visible when representing the DEM as a 3-D surface, producing undesirable artifacts.

Actually the fact that the AMLE cannot interpolate slopes comes from the axiomatic approach that leads to this model. Hence, in order to avoid these artifacts, we have to go back to our assumptions and do the necessary adaptations. First, we will require our operator $\mathrm{E}$ to interpolate not only boundary data, but also boundary slopes. Put another way, our operator will take the form $E(\Omega, \varphi, \psi): \Omega \rightarrow \mathbb{R}$ where $\varphi=\left.u\right|_{\partial \Omega}$ is the boundary data as usual, and $\psi=\partial u /\left.\partial n\right|_{\partial \Omega}$ is the derivative of $\mathrm{u}$ in the direction of the inner normal to $\Omega$. The stability (P1) and invariance properties (P4)-(P8) can be trivially generalized to the new notation. The other two axioms will require, however certain adaptations.

First, once we require $E$ to interpolate slopes as well, (P2) does no longer make sense. A reasonable generalization is to require an order for the slopes as well as for the data

$\left(\mathbf{P 2}^{\prime}\right)$ If $\varphi_{1} \leq \varphi_{2}$ and $\psi_{1} \leq \psi_{2}$ on $\partial \Omega$ then $E\left(\Omega, \varphi_{1}, \psi_{1}\right) \leq$ $E\left(\Omega, \varphi_{2}, \psi_{2}\right)$.

Similarly, since it is not possible to interpolate data and slopes with second-order operators, we will require at least fourthorder. Hence, we have to modify the regularity property as follows:

$\left(\mathbf{P 3}^{\prime}\right)$ If $u(x)$ is a fourth order polynomial with coefficients $c_{0}, \ldots, c_{n}$ then

$$
\begin{array}{r}
\lim _{r \rightarrow 0} \frac{E\left(u,\left.u\right|_{D\left(x_{0}, r\right)},\left.\frac{\partial u}{\partial n}\right|_{D\left(x_{0}, r\right)}\right)\left(x_{0}\right)-u\left(x_{0}\right)}{r^{4}} \\
=F\left(c_{n}, \ldots, c_{0}, x_{0}\right) .
\end{array}
$$

Among fourth-order differential operators the biharmonic operator

$$
\Delta^{2}=\frac{\partial^{4}}{\partial x^{4}}+2 \frac{\partial^{2}}{\partial x^{2} \partial y^{2}}+\frac{\partial^{4}}{\partial y^{4}}
$$

is well-known, and its application to surface interpolation dates back to [12]-[14]. The interpolating operator $u=E(\Omega, \varphi, \psi)$ consists of solving the biharmonic problem

$$
\left\{\begin{array}{l}
\left.\Delta^{2} u\right|_{\Omega}=0 \\
\left.u\right|_{\partial \Omega}=\varphi \\
\left.\frac{\partial u}{\partial n}\right|_{\partial \Omega}=\psi
\end{array}\right.
$$

and is known to have a unique solution under quite general conditions on $\Omega, \varphi$, and $\psi$.

This interpolator was called thin-plate spline because it closely models the shape taken by a thin-metal plate when forced to the given boundary conditions. In such a situation, the plate minimizes its bending energy

$$
J(u)=\iint_{\Omega}\left(\frac{\partial^{2} u}{\partial x^{2}}\right)^{2}+2\left(\frac{\partial^{2} u}{\partial x \partial y}\right)^{2}+\left(\frac{\partial^{2} u}{\partial y^{2}}\right)^{2} d x d y
$$

subject to the boundary conditions $\left.u\right|_{\partial \Omega}=\varphi$ and $\partial u /\left.\partial n\right|_{\partial \Omega}=$ $\psi$. We can easily show that the biharmonic equation (10) is the Euler-Lagrange of the bending energy (11).

This thin-plate spline model has been introduced as an interpolation tool for spatial data in [15] and the references therein. The authors also discuss some variations of the model like the thin-plate spline with tension with the aim of avoiding the oscillations that it may produce. This approach however relies on a tradeoff between Laplacian interpolation (which has zero capacity for points) and biharmonic interpolation, and requires the empirical selection of a tension parameter. So in the rest of this section we shall concentrate on the thin-plate model.

Both from the PDE formulation and from the variational formulation, it is straightforward to verify that the thin-plate spline operator satisfies the invariance properties $(\mathbf{P 4})-(\mathbf{P 8})$. Similarly, the stability property $\left(\mathbf{P 1}^{\prime}\right)$ and the regularity property $\left(\mathbf{P 3}^{\prime}\right)$ are direct consequences of its PDE formulation. The maximum principle (P2'), however, remains more subtle. Actually, [16] and [17] conjectured that it should be true for quite general domains $\Omega$, since Boggio showed that it was true for circular domains. Nevertheless 40 years later a number of counterexamples disproved the conjecture. For instance, [18] showed it is false for rectangular $\Omega$, and [19] and [20] showed it is false for elliptic domains with eccentricity two. In any case, the maximum principle remains valid for small perturbations of circular domains [21].

Unfortunately, there is no equivalent for Theorem 3 either. We do know [23] that for bounded and Lipschitz domains $\Omega$, if $\Delta^{2} u=0$ in $\Omega$ then

$$
\|D u\|_{L^{\infty}(\Omega)} \leq C\|D u\|_{L^{\infty}(\partial \Omega)} .
$$

But the constant $C$ is not necessarily one and depends on the Lipschitz character of the domain $\Omega$.

Furthermore, to the best of our knowledge it is not known whether an interpolation operator exists, which satisfies all of the axioms (P1), $\left(\mathbf{P 2}^{\prime}, \mathbf{P 3}^{\prime}\right)$ and $(\mathbf{P 4})-(\mathbf{P 8})$. If it exists it would be a nice generalization of the AMLE model for slope-interpolating operators. In its absence the thin-plate model comes close by satisfying all but the maximum principle $\left(\mathbf{P 3}^{\prime}\right)$. 


\section{Kriging}

A widely used procedure to interpolate data is kriging, a method originally developed in the framework of geostatistics. We present here a brief account of this method, and give some details on a specific case to be used in our experiments. General references on the subject are [24]-[26]. The surface to interpolate is viewed as a realization of a random field $X$, of which we know values $X\left(x_{1}\right), \ldots, X\left(x_{n}\right)$ at some sites of $\mathbb{R}^{2}$. Through some second order properties (usually to be estimated from the data) of $X$, a "predictor" $\hat{X}$ of $X$ is constructed, defined as a linear combination of the known values of $X$

$$
\hat{X}(x)=\sum_{i=1}^{n} \lambda(x) X\left(x_{i}\right) .
$$

This predictor satisfies two conditions. First, $\hat{X}$ is requested to be unbiased, that is, for all $x$, writing $E$ for the mathematical expectation,

$$
E X(x)=E \hat{X}(x)
$$

The predictor also minimizes the least square error, that is, for all $x$,

$$
\begin{aligned}
& E\left(|\hat{X}(x)-X(x)|^{2}\right) \\
& \quad=\min _{\left\{\lambda_{i}\right\}} E\left(\left|\sum_{i} \lambda_{i} X\left(x_{i}\right)-X(x)\right|^{2}\right) .
\end{aligned}
$$

In other words, $\hat{X}$ is a best linear unbiased predictor (BLUP), a well-known predictor in statistics.

If the field $X$ is second-order stationary (EX is constant and $E(X(x) X(y))=C(x-y)$, for some function $C)$, then $\hat{X}$ is found by inverting a linear system depending only on $\mathrm{C}$ and the data $\left\{x_{1}, \ldots, x_{n}\right\}$ (see [25]). However, and this is quite clear in the case of DEM, $X$ cannot be assumed to be stationary. Therefore, $X$ is assumed to have a "drift," that is $E(X(x))=$ $\sum_{j} \alpha_{j} f_{j}(x)$, for some functions $\left\{f_{j}\right\}$ of $\mathbb{R}^{2}$. The general mathematical framework in which to address the Kriging problem is the one of "intrinsic random functions," a concept first introduced by [27] following the work [28]. Let us briefly introduce this particular type of random fields. We define $P_{k}$ to be the set of polynomials of $\mathbb{R}^{2}$ of degree less or equal to $k$, and an increment with respect to $P_{k}$ to be a set $\left\{\left(\beta_{j}, y_{j}\right)\right\}_{j=1 \cdots m}$ of $\left(\mathbb{R} \times \mathbb{R}^{2}\right)^{m}$, for some integer $m$, such that

$$
\sum_{j=1}^{m} \beta_{j} f\left(y_{j}\right)=0
$$

for all functions $f \in P_{k}$. An intrinsic random function of order $k$ (k-IRF) is a random field such that there exist a function $\gamma: \mathbb{R}^{2} \rightarrow \mathbb{R}$, a "generalized covariance," such that

$$
\operatorname{var}\left(\sum_{i} \beta_{i} X\left(y_{i}\right)\right)=\sum_{i, j} \beta_{i} \beta_{j} \gamma\left(y_{i}-y_{j}\right)
$$

for all increments $\left\{\left(\beta_{i}, y_{i}\right)\right\}$. These fields are completely determined by the generalized covariance $\gamma$, up to a polynomial of degree $k$. Let us write $N_{k}$ for the dimension of the space $P_{k}$, and $\left\{p_{i}\right\}_{i=1} \cdots N_{k}$ for a basis of this space. In this paper, we are interested in particular fields of the form

$$
X(x)=\sum_{i=0}^{N_{k}} a_{i} p_{i}(x)+\epsilon(x),
$$

where $\epsilon$ is a k-IRF such that $E \epsilon=0$, and the $a_{i}^{\prime} s$ are random variables. Fields of the type (16) may be shown to be k-IRF, and are said to have a polynomial drift. The Kriging problem is then expressed, in this particular case, in the same manner as in the stationary case, that is to say that $\hat{X}$ satisfies (14) and (15). It may be shown that this predictor $\hat{X}$ is obtained by inverting a linear system, depending only on $\gamma$ and the data $\left(x_{i}\right)$.

For these fields the following function, defined for $\alpha>0$, is a valid generalized covariance, provided $\operatorname{Int}(\alpha / 2) \leq k$, where $\operatorname{Int}(\alpha)$ is the integer part of $\alpha / 2$

$$
\gamma(x)= \begin{cases}C_{\alpha}|x|^{\alpha}, & \text { if } \frac{\alpha}{2} \notin \mathbb{N} \\ C_{\alpha}|x|^{\alpha} \log (|x|), & \text { if } \frac{\alpha}{2} \in \mathbb{N}\end{cases}
$$

where $C_{\alpha}$ are convenient constants depending on $\alpha$. In the rest of this paper, we consider Kriging with fields having such a generalized covariance, and, thus, we do not address the problem of estimating this function from the data. This is primarily motivated by the fact that, as we will see more precisely, this choice of $\gamma$ lead to the same solution of the interpolation problem as with the thin-plates spline method, when choosing $\alpha=2$. We will thus be in a position to compare out results with thin-plates and Kriging interpolators, in a unified manner with various values of $\alpha$ (see Section IV). We further assume that the value of $k$ (the degree of the polynomial drift) is 1 , and thus allows for values $0<\alpha<4$. In this case, and when $\alpha=2, \hat{X}$ (actually the realization of $\hat{X}$ corresponding to the realization of $X$ ) may be shown to be a solution of the biharmonic problem (10), on $\mathbb{R}^{2}$, with limit conditions [keeping the same notations as in (10)] $u\left(x_{i}\right)=\phi\left(x_{i}\right)$, for $i=1, \ldots, n$, and $\partial u / \partial n=0$ at infinity (see [29] and [30]). Numerically, the equivalence may be seen by considering radial functions as discussed in Appendix C. Explicitly, we write $\mathbf{g}=\left(\gamma\left(x_{0}-x_{1}\right), \ldots, \gamma\left(x_{0}-x_{n}\right)\right), \mathbf{p}=$ $\left(p_{1}\left(x_{0}\right), \ldots, p_{N_{k}}\left(x_{n}\right)\right), \mathbf{z}=\left(Z\left(x_{1}\right), \ldots, Z\left(x_{n}\right)\right)^{T}$, and define the matrices $\mathbf{G}=\left(\left(\gamma\left(x_{i}-x_{j}\right)\right)\right)$ and $\mathbf{P}=\left(\left(p_{j}\left(x_{i}\right)\right)\right){ }^{4}$ Then, the value of the predictor at a point $x_{0}$ is given by (see [25]):

$$
\hat{Z}\left(x_{0}\right)=\mathbf{g a}+\mathbf{p b}
$$

where $\mathbf{a}$ and $\mathbf{b}$ are column vectors such that

$$
\begin{array}{r}
\mathbf{G a}+\mathbf{P b}=\mathbf{z} \\
\mathbf{P}^{T} \mathbf{a}=0 .
\end{array}
$$

When $k=1$ and $\gamma$ is given by (17) with $\alpha=2$, these are the same equations as in the resolution of the thin-plate problem

\footnotetext{
${ }^{4}$ Let us recall that in our experiments we only consider 1-IRFs, so $k=$ $1, N_{k}=3$, and the basis of $P_{k}$ is made up of $p_{1}\left(\begin{array}{l}x \\ y\end{array}\right)=1, p_{2}\left(\begin{array}{l}x \\ y\end{array}\right)=x$, and $p_{3}\left(\begin{array}{l}x \\ y\end{array}\right)=y$.
} 

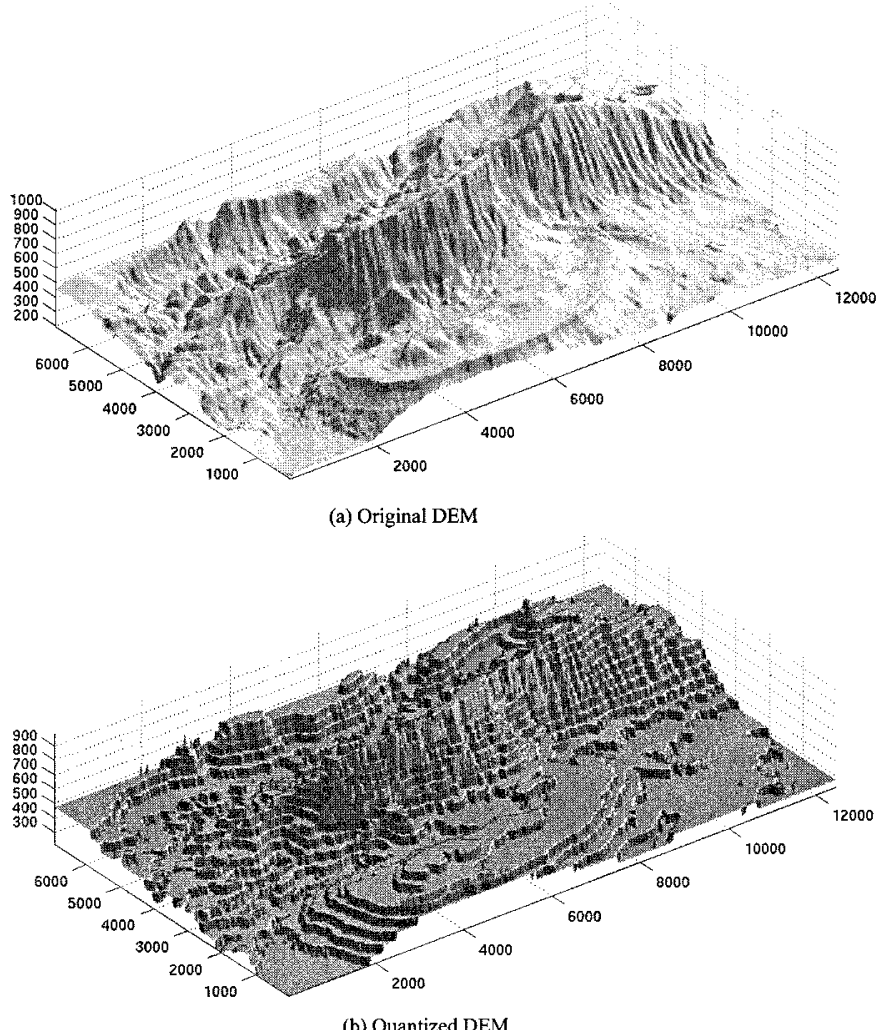

Fig. 1. (a) Elevation model used throughout the experiments. It represents a $12.4 \times 6.9 \mathrm{~km}$ area around Mount Sainte Victoire with heights values ranging between 190 and $1011 \mathrm{~m}$. The horizontal sampling is $30 \mathrm{~m}$, whereas the vertical precision is about $2 \mathrm{~m}$ standard deviation. In all figures, all axes values are expressed in meters, but the vertical axis has been stretched by a factor of three with respect to the real aspect ratio in order to better visualize the irregularities of the terrain. (b) In the quantized DEM light gray colors represent known data points whereas dark gray colors represent unknown points to be interpolated. It has been obtained from the original by only keeping level-curves at regular intervals of 50-m height.

through radial functions like in [31], since in that case the variogram $\gamma(x)=|x|^{2} \log (x)$ is the fundamental solution of the biharmonic equation $\Delta^{2} \gamma=\delta$. More generally, when $\alpha \neq 2$, the kriging solution may be obtained by using $\gamma$ as a radial function.

\section{EXPERIMENTS}

The figures and tables in this paper show some results of interpolation by both the AMLE model, and the classical thin-plates and Kriging models discussed in this paper. To illustrate these methods we chose the elevation model shown in Fig. 1(a) (where height is represented as a three-dimensional illuminated surface). This is a $12.42 \times 6.9 \mathrm{~km}$ DEM around Mount Sainte Victoire where each pixel represents a $30 \times 30 \mathrm{~m}$ patch. Height values vary between 190 and $1011 \mathrm{~m}$ and have a precision of about $2 \mathrm{~m}$. As explained in Section III C, we chose a Kriging model with generalized covariance given by (17), so that thinplate interpolation corresponds mathematically to the case $\alpha=$ 2 . The use of radial functions enables these two methods to agree also from the numerical point of view.

As a first experiment we quantized this elevation model by keeping only the level curves at regular intervals of 50-m height,

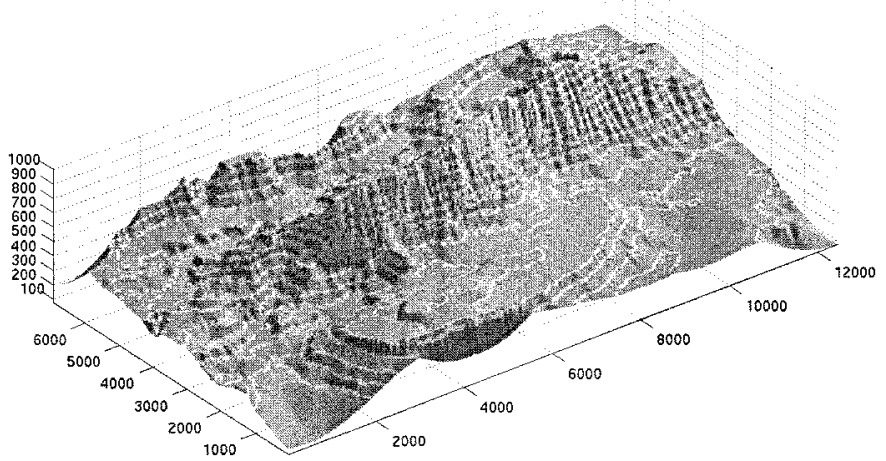

(a) AMLE interpolation

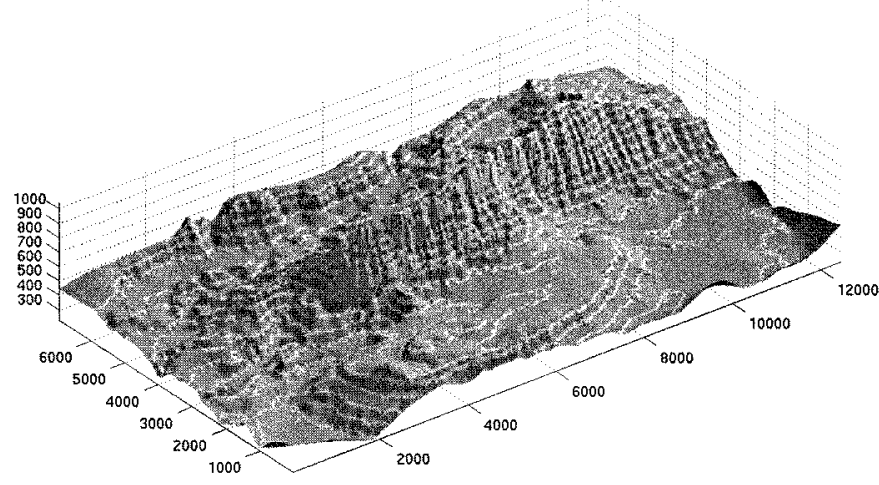

(b) Thin-plate interpolation

Fig. 2. Interpolations of the quantized elevation model from Fig. 1(b). Light gray colors represent known data points whereas dark gray colors represent interpolated points. Observe how AMLE produces certain flat regions and slope discontinuities, but better preserves ridges and avoids some oscillations produced by the thin-plate spline.

as a way to simulate the kind of data that can be obtained from scanning nondigital cartography [see Fig. 1(b)]. Then, we try to reconstruct the original DEM by interpolation using the AMLE model [see Fig. 2(a)] and the thin-plate models [see Fig. 2(b)].

As a second experiment we simulated the kind of interpolation domains that arise in DEMs produced from stereoscopic views. We present four examples: in the first one, we eliminate the top of a mountain [Fig. 3(a)], in the following two, we eliminate a closed region on the side of it, Fig. 3(b) and (c), and in the last one we eliminate a flat region, Fig. 3(d). The corresponding interpolations by AMLE and kriging models can be observed in Figs. 4-7. In Table I, we display the $L^{2}$ distances between original and interpolated DEMs for the different methods.

In these experiments, we can observe how (unlike the AMLE model), the thin-plate and Kriging models allow to interpolate slopes, and produce $C^{1}$ (i.e., with one continuous derivative) interpolants across imposed level curves. Thanks to this ability they allow to roughly recreate the mountain top in Fig. 4(c).

On the other hand, they are somehow much more diffusive than the AMLE model, producing too smooth reliefs. For instance in examples b and c [Figs. 5(a) and 6(a)] we see how the AMLE model better preserved the ridges. In the second case AMLE is better both visually and in terms of RMS error, whereas in the first case AMLE is visually better despite a slightly larger 


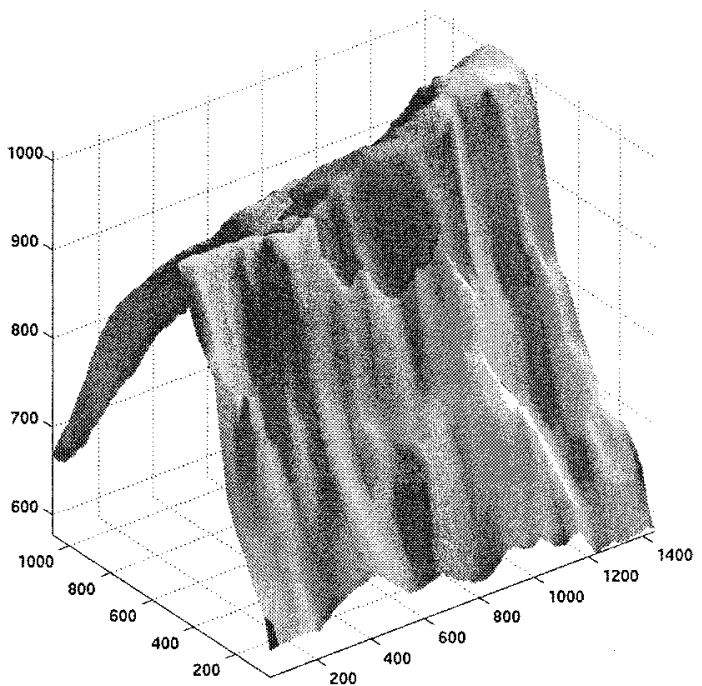

(a) Original DEM for example a

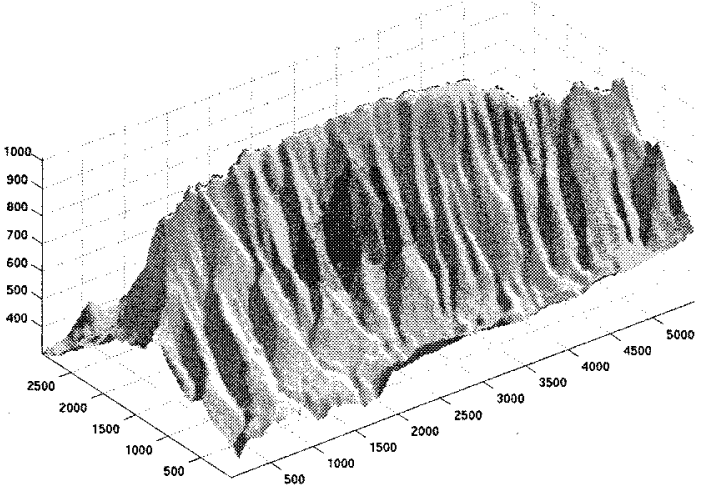

(c) Original DEM for example c

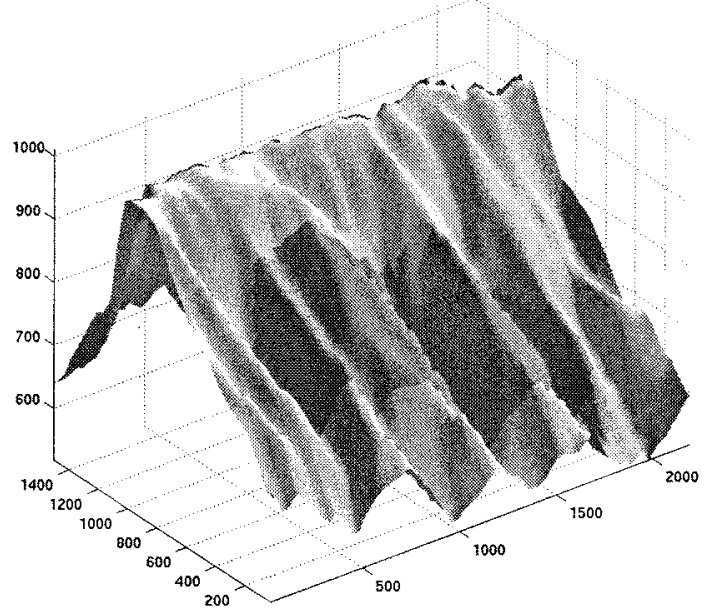

(b) Original DEM for example $b$

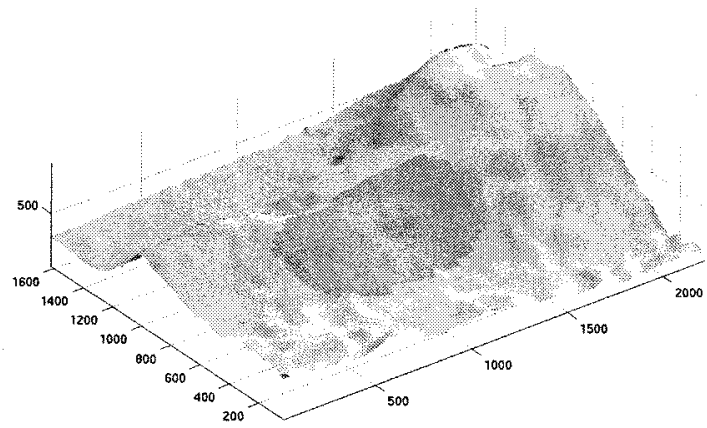

(d) Original DEM for example d

Fig. 3. Interpolation domains used for the second experiment. Light gray colors represent known data points whereas dark gray colors represent unknown points to be interpolated.

RMS error. A similar observation can be made about the last example in Fig. 7(a), where AMLE is visually better despite a slightly larger RMS error. In general, the AMLE produces a better result when the region to be interpolated is entirely contained on one side of the mountain or in a roughly flat region. In those cases the relative slope variations around the boundary are not important so it is not necessary to actually impose them: the average slope is implicit in the boundary data.

Furthermore the fact that thin-plate and kriging do not satisfy a maximum principle means that they are less safe than the AMLE model, in the sense that it can create unnatural oscillations. With respect to Kriging, we can observe how a larger value of $\alpha$ can be used to reduce the diffusive behavior of the thin-plate $(\alpha=2)$, and make it better preserve ridges, although not as good as the AMLE. However, larger values of $\alpha$ lead also to increasingly ill-conditioned systems and to even more unnatural oscillations, as it can be observed for instance in Fig. 5(d).

\section{DISCUSSION AND FURTHER WORK}

In this paper we propose the AMLE as a new method for interpolating digital elevation models and explore its relationship to previously used methods.

Unlike other methods that have been proposed in the geoscience literature, the AMLE satisfies a maximum principle, which ensures that it does not create oscillations. We also showed through experiments that it also preserves ridges much better than Kriging and thin-plate methods, which are much more diffusive. Even though Kriging for high values of $\alpha$ comes a bit closer to the AMLE (in terms of ridge preservation), it's exactly when $\alpha$ is high that Kriging produces the most undesirable oscillations. Also in terms of computational efficiency it can be faster than Kriging, depending on the data.

In the case of interpolation of iso-level lines AMLE can be interpreted as iterated geodesic distance transformations, which explains why it shares some qualitative properties with this method. 


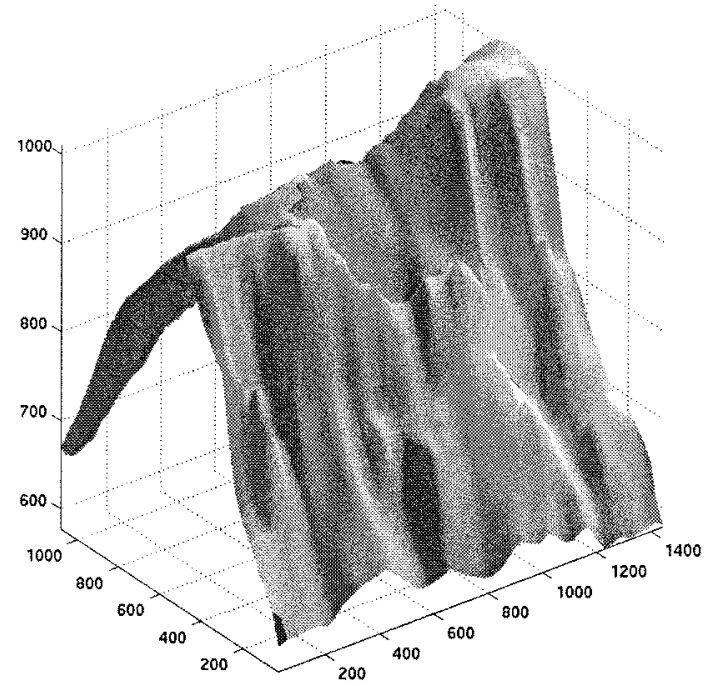

(a) AMLE interpolation of figure $3 \mathrm{a}$

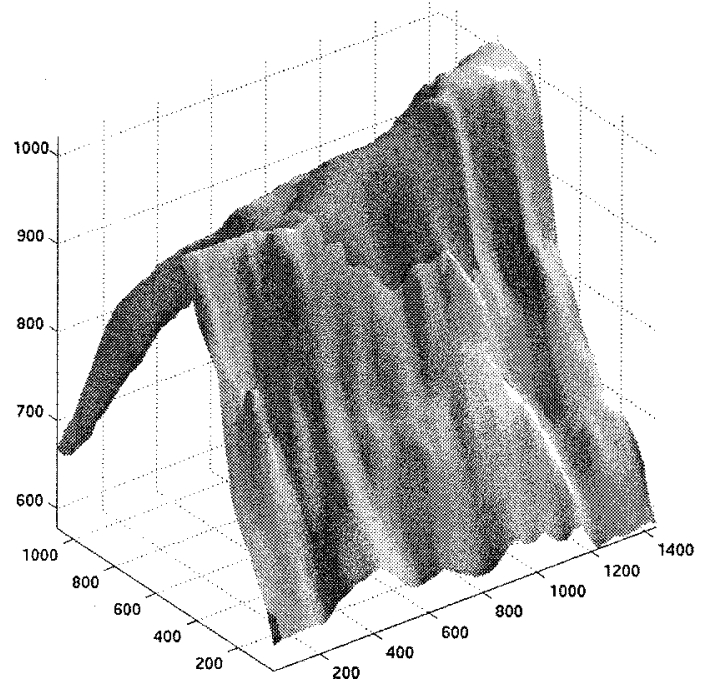

(c) Kriging (and thin plates) interpolation of figure 3a, $\alpha=$

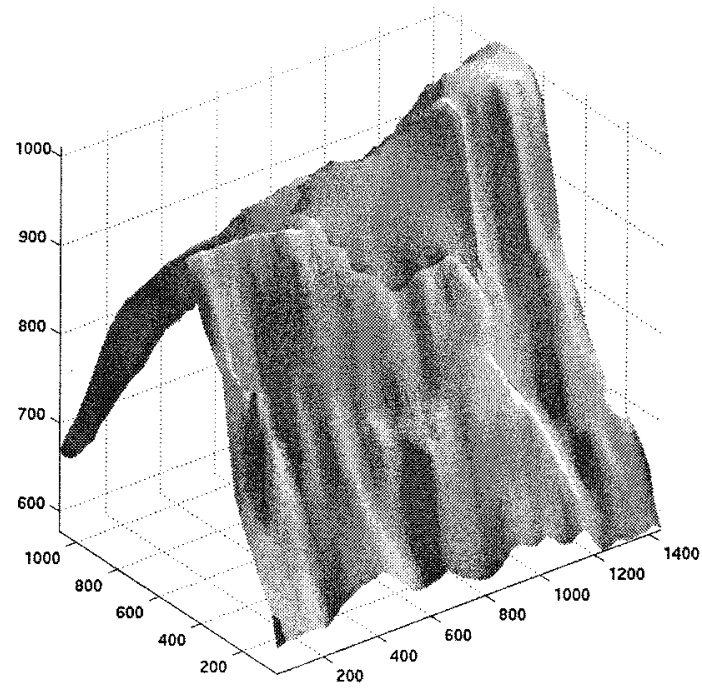

(b) Kriging interpolation of figure $3 \mathrm{a}, \alpha=1$

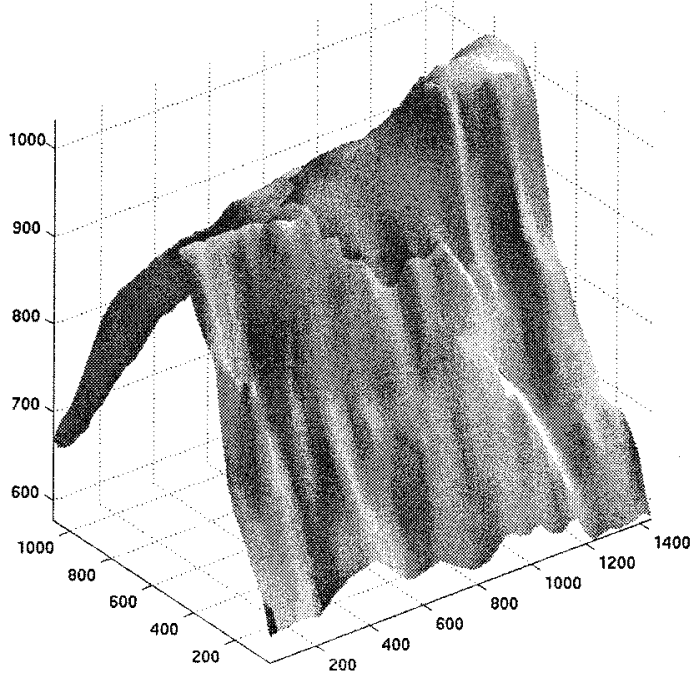

(d) Kriging interpolation of figure $3 a, \alpha=3$

Fig. 4. Interpolations of the first example, Fig. 3(a). Light gray colors represent known data points whereas dark gray colors represent interpolated points.

The major disadvantage of AMLE is the fact that it cannot interpolate slopes as well as values. For this purpose we proposed a reasonable generalization to slope-interpolating operators of the axiomatic approach that leads to the AMLE model. None of the currently known methods satisfies all of these axioms. The thin-plate method comes closest by satisfying all but the maximum principle, hence it may produce unnatural oscillations. The search for a (possibly nonlinear) fourth-order differential operator satisfying all of the proposed axioms remains an interesting problem for future research, since it might lead to a slope-interpolating operator with all the advantages of AMLE.

In the absence of such a generalized interpolator, we would propose a combined use of AMLE and Kriging or thin-plate methods. This could be done in a way which allows to use AMLE whenever fixing slope values is not necessary, and Kriging otherwise. This would require for each connected com- ponent of the interpolation domain, either a human decision, or an automatic procedure which first applies AMLE and then keeps it if the slope discontinuities it creates on this connected component are below a certain threshold, or substitutes it by Kriging. Another possibility consists of adding to the AMLE interpolation, a factor $(\beta \leq 1)$ times the solution of thin-plate interpolation with zero boundary condition, and slope boundary condition equal to the slope discontinuity introduced by AMLE. This would probably produce a quasislope-interpolating operator which better preserves ridges.

\section{APPENDIX \\ ALGORITHMIC COMPLEXITY}

In this section we briefly discuss the complexity of the algorithms we used for computing the different interpolation methods proposed. The computing times we needed on a 


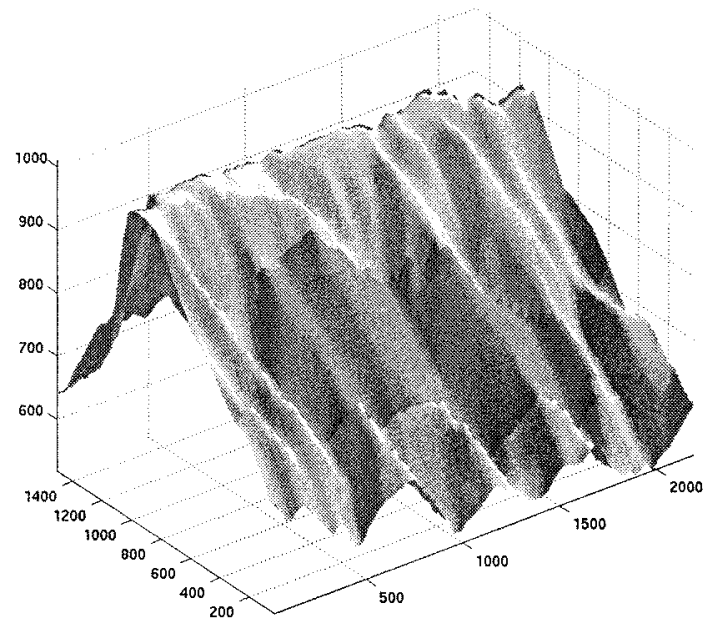

(a) AMLE interpolation of figure $3 b$

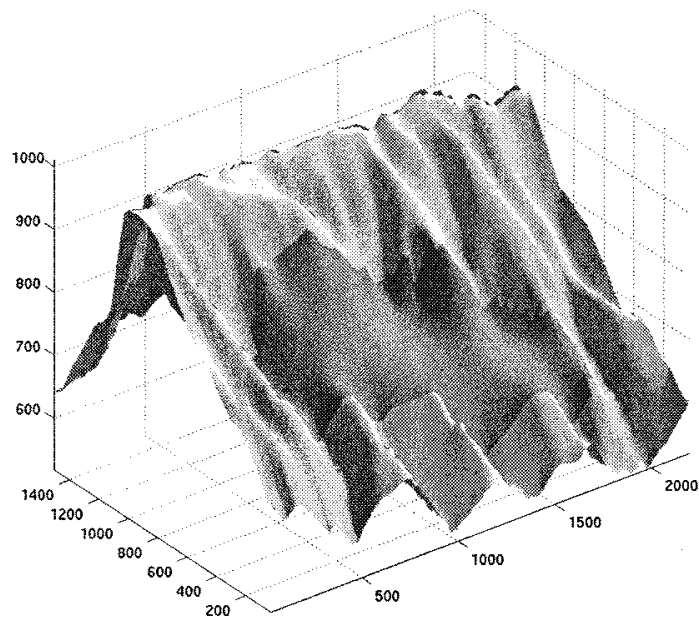

(c) Kriging (and thin plates) interpolation of figure $3 \mathrm{~b}$, $\alpha=2$

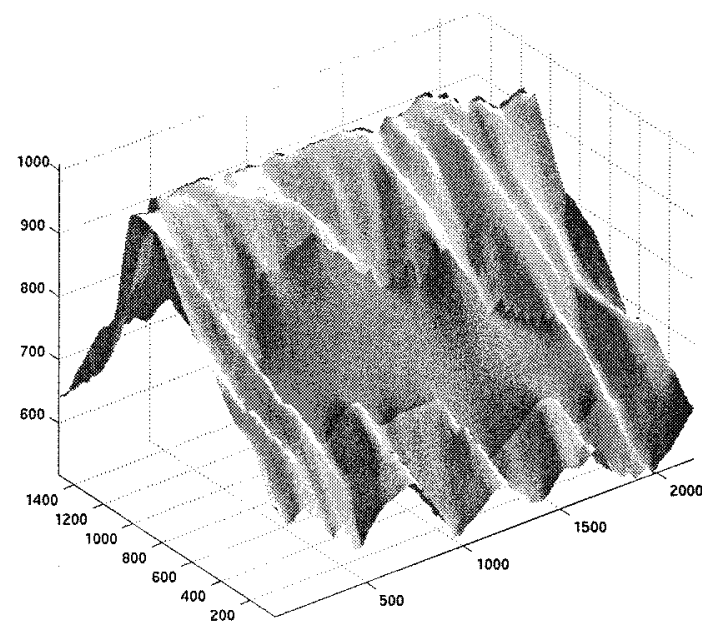

(b) Kriging interpolation of figure $3 \mathrm{~b}, \alpha=1$

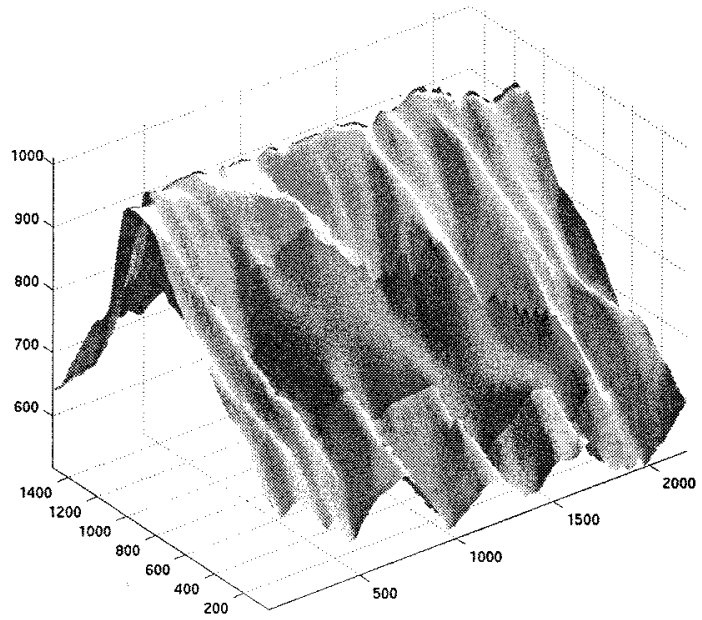

(d) Kriging interpolation of figure $3 \mathrm{~b}, \alpha=3$

Fig. 5. Interpolations of the second example, Fig. 3(b). Light gray colors represent known data points whereas dark gray colors represent interpolated points. Observe how AMLE better preserves the ridges. Among Kriging methods, ridges are best preserved by the highest values of $\alpha$, but at the cost of more oscillations than lower values of $\alpha$ and AMLE. Lower values of $\alpha$, on the other hand are much too diffusive.

SUN UltraSparc processor at $336 \mathrm{MHz}$ to run the examples in Table I are given in Table II. These figures should be taken with care when comparing methods, because our implementations of these algorithms are not optimal in all cases, and they are implemented in different languages (C for AMLE and Matlab for thin-plate and Kriging).

In the following paragraphs, we give an indication of the computational complexity as well as some possible improvements in their implementations. This should be more useful to compare the performance of the different methods.In all cases we assume that the region $\Omega$ to be interpolated consists of $N$ grid-points, and that its boundary $\partial \Omega$ is composed of $L$ grid-points. Furthermore, we call $M$ the number of interpolation constraints, which in the case of AMLE and distance transforms is $M=L$ and in the case of thin-plate and Kriging is usually in the range $M \in[L, 2 L]$. This is due to the fact that in order to impose slopes on the boundary, what we do in practice is impose the values on a dilated boundary, which is normally composed of about $2 L$ points, except if data is not available on the dilated boundary as in the case of contour-line interpolation.

\section{A. AMLE Using Finite Differences}

In [32] a consistent finite-difference scheme was proposed for solving the AMLE. It consists of iterating

$$
\frac{\partial u}{\partial t}=D^{2} u\left(\frac{D u}{|D u|}, \frac{D u}{|D u|}\right)
$$

with the same boundary condition $u(t, x)=\varphi(x), \quad(t, x) \in$ $(0,+\infty) \times \partial \Omega$ and any initial condition $u(0, x)=$ $u_{0}(x), \quad x \in \Omega$ until a steady-state is found (for $t \rightarrow \infty$ ), which satisfies the AMLE equation $D^{2} u(D u, D u)=0$ up to a certain tolerance. Each time step is discretized in a semi-implicit manner, using one iteration of nonlinear over-relaxation to solve it. The global complexity is then $n N C$ flops, where $n$ is the number of iterations needed to reach the steady-state, 


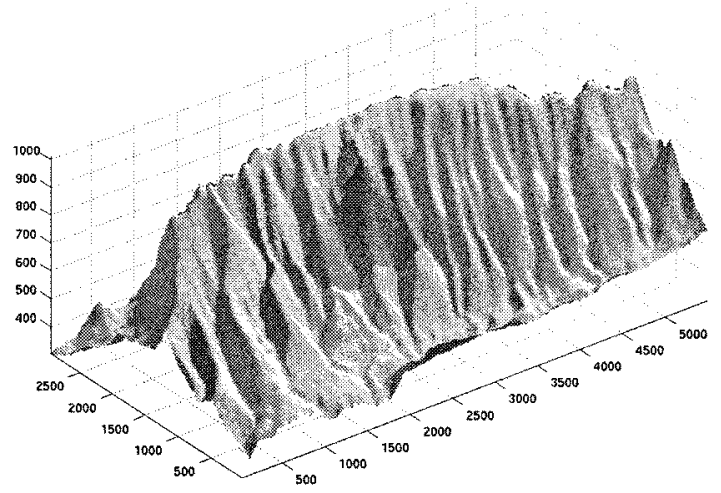

(a) AMLE interpolation of figure $3 \mathrm{c}$

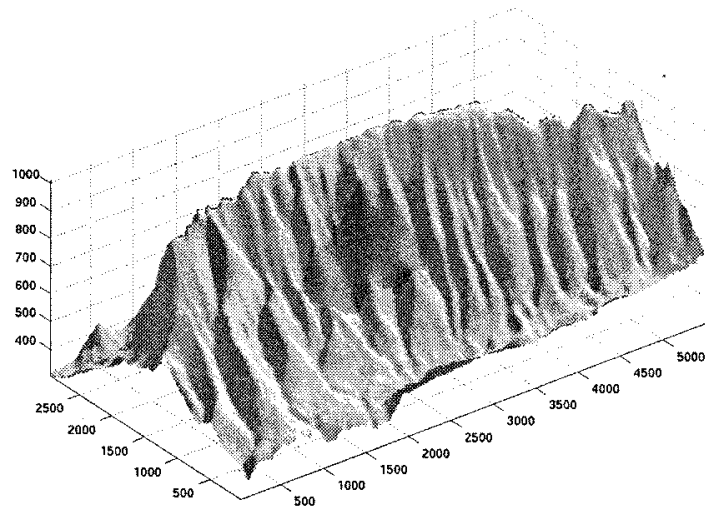

(c) Kriging (and thin plates) interpolation of figure $3 \mathrm{c}, \alpha=2$

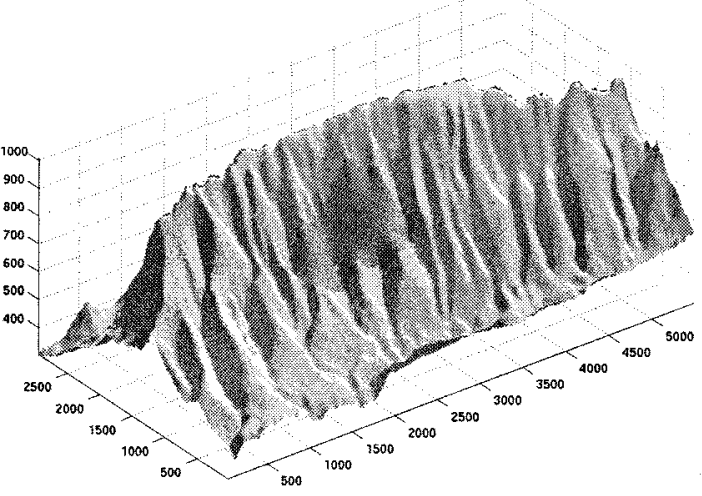

(b) Kriging interpolation of figure $3 \mathrm{c}, \alpha=1$

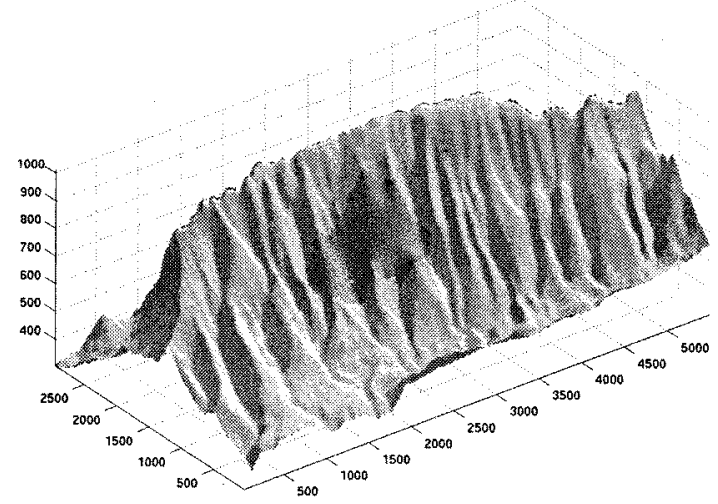

(d) Kriging interpolation of figure $3 c, \alpha=3$

Fig. 6. Interpolations of the third example, Fig. 3(c). Light gray colors represent known data points whereas dark gray colors represent interpolated points. Observe how AMLE preserves the ridges remarkably better than Kriging.

and $C \approx 50$ is the constant number of floating point operations per point and iteration. In our examples, $N \approx 200$ and we need about $n=100$ iterations to achieve a precision of two decimal digits, and about $n=200$ iterations for a precision of four digits. Total running times for these examples are 0.2 and $0.3 \mathrm{~s}$, respectively, on a Sun SPARC workstation.

The number of iterations is highly dependent though on the size of the maximal gap in the interpolation domain. In a bigger example with $N \approx 100000$, we need about $n=3000$ iterations (10 min) for a precision of two decimal digits.

Nevertheless, our implementation of the AMLE can be significantly accelerated for domains with large gaps by means of a multigrid algorithm, and by substituting nonlinear over-relaxation by a variant of the preconditioned conjugate gradient method.

\section{B. AMLE Through Iterated Geodesic Distance Transforms}

As we observed in Section III-A, in the special case of contour-line interpolation, the AMLE can be obtained by iterated geodesic distance transform interpolations. The complexity of one iteration is $C N$ flops, where $C$ is a small constant, if we compute the weighted geodesic distances by a propagation algorithm like the one in [33], which visits each pixel just a few number of times. The total complexity of AMLE through geodesic distances is then $n C N$ flops, but the number of iterations $n$ is here much smaller than in the case of finite differences.

\section{Thin-Plate and Kriging Through Radial Basis Functions}

Kriging and thin-plate interpolations can be both computed, as explained in Section III-C, by solving (19) in order to find the Kriging coefficient vectors $\mathbf{a}$ and $\mathbf{b}$ [which takes $1 / 3(M+3)^{3}$ flops in the case of 1-IRF, where $N_{k}=3$ ], and then using equation (18) to find the value of the interpolant at each point [which takes $(M+3) N$ flops]. So, the total complexity is $1 / 3(M+$ $3)^{3}+(M+3) N$ flops. This is much faster than AMLE when $M$ is small with respect to $N$ (a roughly circular interpolation domain for instance), but much slower when $M$ is large, which happens, e.g., when the interpolation domain has a very irregular boundary.

\section{Thin-Plate Through Finite Differences}

In the special case of $\alpha=2$ Kriging coincides with the thin-plate model and we can use a finite difference method to solve it. The spatial discretization of the biharmonic equation with boundary conditions (10) leads to a sparse (block-banded) $(N+M) \times N$ linear system with 21 bands (one for each point in the $5 \times 5$ stencil used to discretize the bilaplacian operator). To solve this system we used either a multigrid approach, or a 


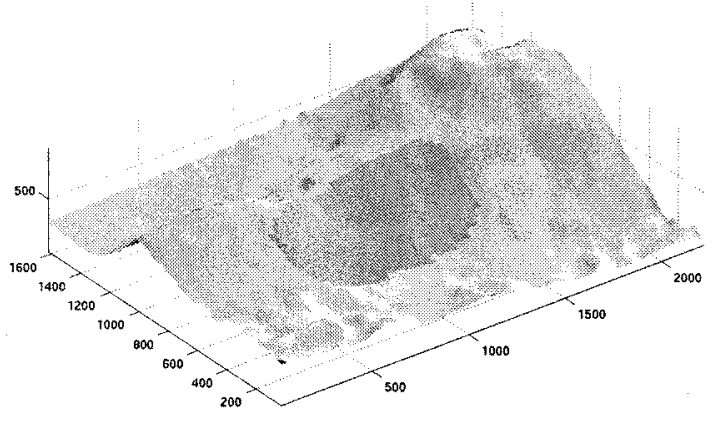

(a) AMLE interpolation of figure $3 \mathrm{~d}$

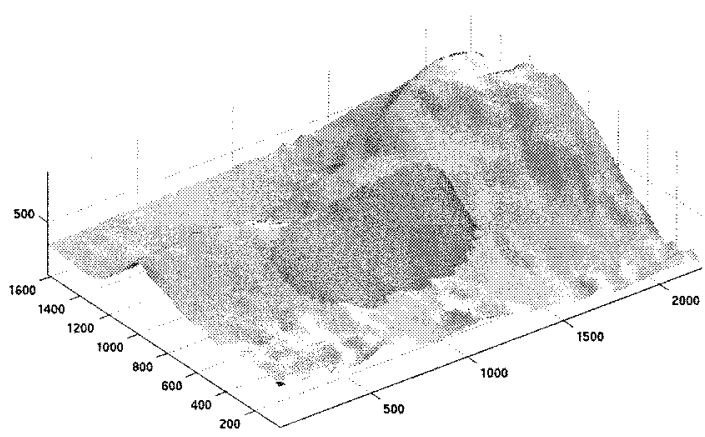

(c) Kriging (and thin plates) interpolation of figure $3 \mathrm{~d}, \alpha=2$

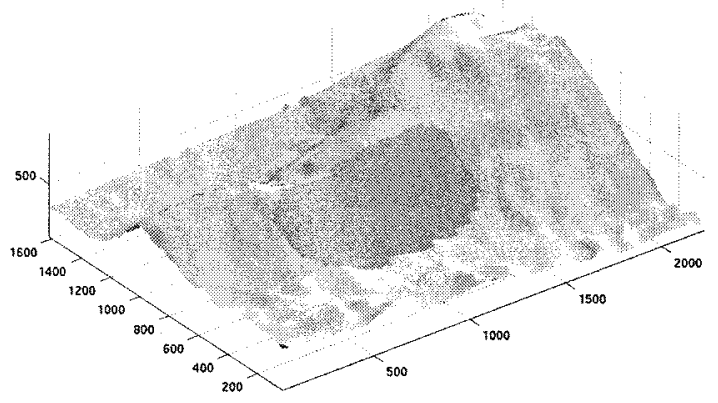

(b) Kriging interpolation of figure $3 \mathrm{~d}, \alpha=1$

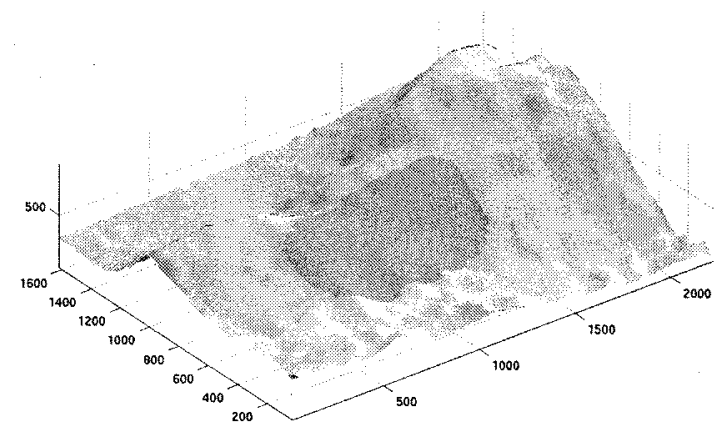

(d) Kriging interpolation of figure $3 \mathrm{~d}, \alpha=3$

Fig. 7. Interpolations of the third example, Fig. 3(d). Light gray colors represent known data points whereas dark gray colors represent interpolated points.

TABLE I

$L^{2}$ Distances BETWEen ORIGINALS AND INTERPOlated FOR EXAMPLES a-d, FIG. 3 (All VALUeS ARE EXPRESSED IN METERS)

\begin{tabular}{c|c|c|c|c}
\hline method & example a & example b & example c & example d \\
\hline AMLE & 52.88 & 25.21 & 25.03 & 10.64 \\
Kriging, $\alpha=1$ & 26.57 & 28.22 & 37.22 & 7.15 \\
Kriging, $\alpha=2$ (thin plates) & 16.72 & 24.84 & 37.00 & 5.41 \\
Kriging, $\alpha=3$ & 20.57 & 22.40 & 32.41 & 5.81 \\
Kriging, $\alpha=3.9$ & 27.94 & 25.90 & 29.01 & 8.75 \\
\hline
\end{tabular}

TABLE II

Computational Resources for the EXAMPLeS IN TABLE I. All VAlues ARE EXPRESSED IN SECONDS (AND $M$ FlopS). AT THE END We INDICATE the Size of the Problem in Terms of $N$ (Size of Region to BE INTERPOLATED IN PIXELS) AND $M$ (SIZE OF REGION BOUNDARY, I.E., THE NUMBER OF INTERPOLATION CONSTRAINTS)

\begin{tabular}{c|c|c|c|c}
\hline method & example a & example b & example c & example d \\
\hline AMLE & $0.2(1.97)$ & $1.1(15.36)$ & $3.9(20.49)$ & $1.1(11.77)$ \\
Kriging, $\alpha=1$ & $0.14(2.78)$ & $1.84(22.78)$ & $1.74(20.58)$ & $0.69(9.45)$ \\
Kriging, $\alpha=2$ (thin plates) & $0.15(2.89)$ & $2.23(23.47)$ & $1.97(21.72)$ & $0.86(9.78)$ \\
Kriging, $\alpha=3$ & $0.16(2.78)$ & $2.11(22.78)$ & $1.74(20.58)$ & $0.87(9.45)$ \\
Kriging, $\alpha=3.9$ & $0.17(2.78)$ & $1.94(22.78)$ & $1.20(20.58)$ & $0.82(9.45)$ \\
\hline$N$ & 197 & 768 & 745 & 471 \\
$M$ & 140 & 296 & 285 & 216 \\
\hline
\end{tabular}

preconditioned conjugate-gradient method, with an incomplete LU factorization as a preconditioner. The total complexity is then $n m(N+M)$, where $n$ is the number of iterations of the conjugate gradient algorithm and $m$ is the number of nonzero elements per row in the incomplete LU factorization. In our experiments $m \approx 150$ and $n \ll(N+M)$, typically $n \approx 50$ for a four digit precision. Thus, for large values of $M$ this method performs much better that the radial basis function method derived from Kriging.

\section{ACKNOWLEDGMENT}

The authors would like to thank C. López for providing the elevation data used in this paper, P. Soille for useful discussions, and V. Caselles and C. Sbert for fruitful advice on AMLE and interpolation methods. They would like to specially thank J.-M. Morel for his supervision, encouragement, and useful comments throughout this project.

\section{REFERENCES}

[1] J. Delon and B. Rougé, "Le phénoméne d'adhérence en stéréoscopie dépend du critére de corrélation," in Proc. GRETSI'01, Toulouse, France, Sept.

[2] V. Caselles, J. M. Morel, and C. Sbert, "An axiomatic approach to image interpolation," IEEE Trans. Image Processing, vol. 7, pp. 376-386, Mar. 1998.

[3] G. Aronson, "Extensions of functions satisfying Lipschitz conditions," Arkiv Matematik, vol. 6, no. 28, 1967.

[4] - "On the partial differential equation $u_{x}^{2} u_{x x}+2 u_{x} u_{y} u_{x y}+$ $u_{y}^{2} u_{y y}, "$ Arkiv Matematik, vol. 7, no. 28, 1968.

[5] R. Jensen, "Uniqueness of Lipschitz extension: Minimizing the sup norm of the gradient," Arch. Rat. Mech. Anal., no. 123, pp. 51-74, 1993.

[6] M. G. Crandall, H. Ishii, and P. L. Lions, "User's guide to viscosity solution of second order partial differential equations," Bull. Amer. Math. Soc., vol. 27, no. 1, July 1992. 
[7] I. Capuzzo Dolcetta and P. L. Lions, Eds., "Viscosity solutions and applications," in Lecture Notes in Mathematics. Montecatini Terme, Italy, June 1995, Lectures given at the 2nd session of the Centro Internazionale Matematico Estivo (CIME)

[8] S. Masnou, "Filtrage et Desocclusion d'images par méthodes d'ensembles de niveau," Ph.D. dissertation, Univ. Paris Dauphine, Paris, France, Dec. 1998.

[9] F. Cao, "Absolutely minimizing lipschitz extension with discontinuous boundary data," C. R. Acad. Sci. Paris, vol. 327, pp. 563-568, 1998.

[10] P. Soille, "Spatial distributions from contour lines: An efficient methodology based on distance transformations," J. Vis. Commun. Image Represent., vol. 2, no. 2, pp. 138-150, June 1991.

[11] _ - "Generalized geodesic distances applied to interpolation and shape description," in Mathematical Morphology and Its Applications to Image Processing, J. Serra and P. Soille, Eds. Norwell, MA: Kluwer, 1994.

[12] J. Duchon, "Interpolation des fonctions de deux variables suivant le principe de la flexion des plaques minces," R.A.I.R.O. Anal. Numér., vol. 10, pp. 5-12, 1976

[13] J. Meinguet, "Surface spline interpolation: Basic theory and computational aspects," in Approximation Theory and Spline Functions, S. P. Singh et al., Eds. Dordrecht, Holland: D. Reidel, 1984, pp. 124-142.

[14] R. Franke, "Scattered data interpolation: Test of some methods," Math. Comput., vol. 38, no. 157, pp. 181-200, Jan. 1982.

[15] L. Mitas and H. Mitasova, Spatial Interpolation, 2nd ed. New York: Wiley, 1999, vol. 1, ch. 34, pp. 481-492.

[16] T. Boggio, "Sull'equilibrio delle piastre elastiche incastrate," Rend. Acc. Lincei, vol. 10, pp. 197-205, 1901.

[17] J. Hadamard, "Mémoire sur le problème d'analyze relatif à l'équilibre des plaques élastiques incastrées," Mémoires Présentées par Divers Savants a l'Académie des Sciences, vol. 33, pp. 1-128, 1908

[18] C. V. Coffman, "On the structure of solutions of $\delta^{2} u=\lambda u$ which satisfy the clamped plate condition on a right angle," SIAM J. Math. Anal., vol. 13, pp. 746-757, 1982.

[19] P. R. Garabedian, "A partial differential equation arising in conformal mapping," Pacific J. Math., vol. 1, pp. 485-524, 1951.

[20] Partial Differential Equations. New York: Chelsea, 1986, p. 275

[21] C. Grunau and G. Sweers, "Positivity for perturbations of polyharmonic operators with dirichlet boundary conditions in two dimensions," Math. Nachr, vol. 179, pp. 89-102, 1996

[22] H. Hendenmalm, "An Hadamard maximum principle for the bilaplacian on hyperbolic manifolds," Journées Équations aux dérivées partielles, Saint-Jean-de-Monts, June 1999, GDR 1151 (CNRS).

[23] J. Pipher and G. Verchota, "A maximum principle for biharmonic functions in Lipschitz and $c^{1}$ domains," Comment. Math. Helvetici, vol. 68, pp. 385-414, 1993

[24] G. Matheron, "La théorie des variables régionalisées, et ses applications," Les Cahiers du Centre de Morphologie Mathematique de Fontainebleau, vol. 5, 1971

[25] N. Cressie, Statistics for Spatial Data, revised ed. New York: Wiley, 1993.

[26] A. G. Journel and C. J. Huijbregts, Mining Geostatistics. London, U.K.: Academic, 1978

[27] G. Matheron, "The intrinsic random functions and their applications," Adv. Appl. Prob., vol. 5, pp. 439-468, 1973.

[28] I. M. Gel'fand and N. Y. Vilenkin, Generalized Functions. New York: Academic, 1964, vol. 4

[29] G. Matheron, "Splines and kriging; their formal equivalence," in Down-to-Earth Statistics: Solutions Looking for Geological Problems, D. F. Merriam, Ed. Syracuse, NY: Syracuse University Geology Contributions, 1981, pp. 77-95.

[30] J. T. Kent and K. V. Mardia, "The link between kriging and thin-plate splines," in Probability, Statistics and Optimization, F. P. Kelly, Ed. New York: Wiley, 1994, ch. 24.

[31] F. L. Bookstein, "Principal warps: Thin-plate splines and the decomposition of deformations," IEEE Trans. Pattern Anal. Machine Intell., vol. 11, pp. 567-585, June 1989.
[32] V. Caselles, J. M. Morel, and C. Sbert, "An axiomatic approach to image interpolation,” IEEE Trans. Image Processing, vol. 7, pp. 376-386, Mar. 1998.

[33] P. Soille, "Morphologie Mathématique: du relief à la dimensionalité-Algorithmes et méthodes," Ph.D. dissertation, Univ. Catholique de Louvain-la-Neuve, Belgium, 1992.

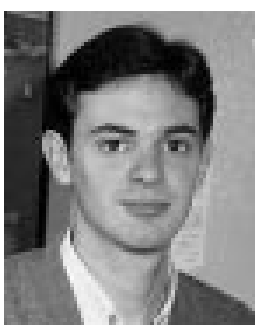

Andrés Almansa received the Engineer degree in computer science from the University of the Republic of Uruguay (UR), Montevideo, in 1995 and the M.Sc. degree in computer science jointly from UR and the Royal Institute of Technology, Stockholm, Sweden, in 1998.

He is with ENS Cachan, France, working for the French Space Agency (CNES) on satellite image acquisition and digital elevation models from stereo pairs. In 1998, he became an Adjunct Professor with the School of Engineering, UR, where he has been teaching computer science and numerical analysis, and working on fingerprint image processing, since 1993.

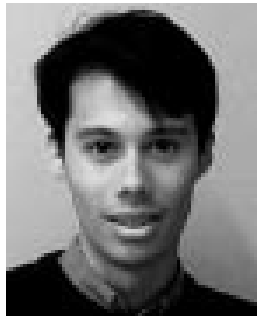

Frédéric Cao graduated from École Polytechnique, Paris, France in 1995. He received the Ph.D. dissertation in applied mathematics in the École Normale Supérieure de Cachan in 2000.

His research subjects are some applications of partial differential equations to image processing and particularly level set methods. He worked with the French Ministry of Defense in 2000-2001, before joining INRIA-Rennes, Toulouse, France, in 2001.

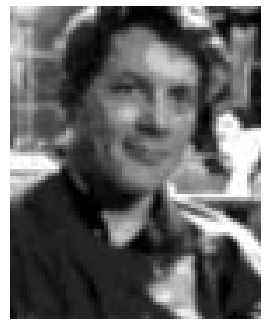

Yann Gousseau graduated from the École Centrale de Paris, France, in 1995. He received the Ph.D. degree in applied mathematics at the Université de Paris 9 Dauphine in 2000

His research interests are in the statistics of natural images and mathematical morphology. He worked as a Postdoctoral Researcher with École Normale Supérieure de Cachan in 2000-2001, before joining École Nationale Supérieure des Télécommunications, Paris, in 2001.

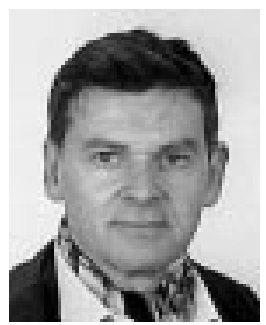

Bernard Rougé received the Ph.D. degree in probabilities from Institut Henry Poincaré, Paris, France, in 1975, and the Habilitation à diriger des Recherches degree from Université de Paris-Dauphine in 1997.

$\mathrm{He}$ is currently an Engineer with the French Space Agency (CNES), and Researcher with CMLA (CNRS UMR 8536), ENS Cachan, France. His main research interest is a global approach to the quality of space imagery (sampling, restoration, and compression). 